\title{
Antoine und Robert de Fevin: Ihre Messen in Brünner Manuskript BAM1
}

\section{Antoine and Robert de Fevin: their Masses in the Brno Manuscript BAM1}

\author{
Hana Studeničová / 362251@mail.muni.cz \\ Ústav hudební vědy, Filozofická fakulta, Masarykova univerzita, Brno, CZ
}

\begin{abstract}
The recently discovered Brno manuscript BAM1 (Brno city archive. Manuscript collection, sig. 15/4) brings further understanding into the field of practice of 16th century music in Brno and introduces another research opportunities. The manuscript contains three polyphonic masses of less known composers Antoine and Robert de Fevin, belonging to the third generation of Franco-Flemish polyphonic.

The study presents gathered biographies of both composers, however the major focus belongs to a hypothesis of fraternity of Antoine and Robert, not even excluding the possibility of duplicity of theirs identities. In this study are presented the results of the effected analysis of the composition style of the preserved masses Missa Le vilayn ialoys, Missa Ave Maria and Missa Helas ye suis mari, which is uniquely extant in BAM1 manuscript. These masses reflect the importance of Antoine and Robert de Fevin within the Franco-Flemish polyphony.
\end{abstract}

\section{Key words}

Brno, manuscript BAM1, Franco-Flemish polyphony, Antoine de Fevin, Robert de Fevin, mass 
Die Bedeutung des Schaffens der franko-flämischen Komponisten überschritt die Grenzen dieser Region und beeinflusste musikalisches Geschehen in Mitteleuropa, d. h. auch in den Ländern der Böhmischen Krone. Im vor kurzem gefundenen Brünner Manuskript BAM1 (Sig. 15/4) befinden sich Messen von Josquin Desprez, Heinrich Isaac, Antoine Brumel, Jean Mouton, Antoine und Robert de Fevin, u.a. Die beiden letztgenannten gehören zu wenig bekannten Komponisten der franko-flämischen Polyphonie, genauer gesagt ihrer dritten Generation. ${ }^{1}$ De Fevin werden oft als Brüder genannt, aber es gibt fast keine Beweise, die diese Hypothese unterstützen würden. Ihr Familienname ist die einzige Verbindung zwischen ihnen. Die geringe Menge der biographischen Angaben weist auf die Vermutung hin, dass die Komponisten in der Geschichte der frankoflämischen Polyphonie keine wichtige Rolle spielten. Ihre erhaltenen Kompositionen bestätigen das Gegenteil. In dem Manuskript BAM1 befinden sich drei Messen von Antoine und Robert de Fevin. Das Schaffen von Robert de Fevin vertritt Missa Le vilayn ialoys. Zwei Messen von Antoine de Fevin heißen Missa Ave Maria und Missa Helas ye suis mari. Die zweite ist im BAM1 höchstwahrscheinlich einzigartig überliefert.

In diesem Beitrag sollen die bekannten biographischen Daten der beiden Komponisten dargestellt werden. Dank ihnen soll die Frage beantworten werden, ob Antoine und Robert tatsächlich Brüder oder Verwandte sein könnten. Außerdem werden wir uns mit der Hypothese beschäftigen, ob es sich sogar um eine einzige Person handeln könnte. Aus ihrem erhaltenen Schaffen wird die Aufmerksamkeit vor allem auf die Messen gerichtet. Aufgrund der Kompositions- und Stilanalyse von ihren erhaltenen Messen sollen schließlich die Ergebnisse vorgelegt werden.

\section{„Antoine, prêtre, chantre du Roi Louis XII, mort à Blois"}

Die Lebensbahn von Antoine de Fevin ist rätselhaft. Die grundsätzlichen Informationen nicht nur über Antoines Leben, sondern auch über Leben von Robert de Fevin, gehen aus einer Studie über die Familie von Pierre de Fevin hervor. Der französische Forscher De Puisieux ${ }^{2}$ erstellte in dieser Studie einen Stammbaum, aus dem evident ist, dass Antoine aus einer reichen Familie des Gutsbesitzers Pierre de Fevin, der ab dem Jahr 1474 ein Rat in Arras war, stammte. ${ }^{3}$ Als die wichtigste Tatsache scheint das zu sein, dass zwei Söhne von Pierre de Fevin bekannt sind, Philippe und Antoine. Beim Antoines Namen steht in dem Stammbaum kurz: „Antoine, prêtre, chantre du Roi Louis XII, mort à Blois“. Also Antoine war Priester, Sänger bei dem König Ludwig XII. und starb in Blois. Über seinen Tod lässt sich mit Sicherheit sagen, dass er entweder im Dezember 1511 oder spätestens am Beginn des Januar 1512 auf dem königlichen Hof gestorben ist. Es ist un-

1 Aus den praktischen Gründen stimme ich der Gliderung nach EGGEBRECHT, Hans Heinrich. Musik im Abendland: Prozesse und Stationen vom Mittelalter bis zur Gegenwart. München: Piper, 1991, bei.

2 PUISIEUX, G. B. de. Pierre de Févin, chronique artésiem du XVe siécle. In Bulletin de la Société des antiquaires de Picardie, 1909-1910, S. 325-326.

3 Ebenda, S. 325.

4 Ebenda, S. 325. 
gewöhnlich, die Lebensschilderung von Antoine de Fevin mit seinem Tod zu beginnen. Sein Todesdatum ist schließlich eine der wenigen Informationen, die man genau weiß. Demzufolge können wir sein Geburtsdatum abschätzen. Im Januar 1512 verstarb einer von den besten Hofsängern des Königs Ludwig XII., Jean Braconnier (Lourdault). Braconnier starb kurz nach dem 28. Januar 1512. ${ }^{5}$ Dieses genaue Datum ist der Schlüsselbeweis auch bei dem Antoines Todestag. Anlässlich Braconniers Todes schrieb bekannter Dichter Guillaume Crétin eine Elegie. Crétin nennt in der Elegie auch Antoine de Fevin und schätzte ihn hoch. ${ }^{6}$ Mithilfe weiterer erhaltener Quellen ${ }^{7}$ konnte festgelegt werden, dass Antoine de Fevin kurz vor Braconnier starb, d. h. entweder in der letzte Woche im Jahre 1511, oder in ersten Tagen des Jahres 1512.

Andere Situation tritt bei der Festsetzung seines Geburtsdatums an. Es gibt viele Vermutungen, wann Antoine de Fevin geboren sein konnte. Der niederländische Musikwissenschaftler Ben Kahmann, der eine umfangreiche Studie über Antoines Leben und Werk geschrieben hatte, ${ }^{8}$ stellte fest, dass sich die Geburtsdaten bis zu zwanzig Jahren unterscheiden. Die Untergrenze bildet das Jahr 1470, das Johann Gottfried Walter in Musikalisches Lexikon (1732) anführt. ${ }^{9}$ Im Gegenteil dazu schrieb August Wilhelm Ambros, ${ }^{10}$ dass Antoine de Fevin im Jahre 1490 geboren wurde. Im Fall seines kurzen Lebens spielt die Zeitspanne sehr wichtige Rolle. Die Informationen über Phillip, den älteren Bruder von Antoine, können bei der Bestimmung des vorausgesetzten Geburtsdatum behilflich sein. Phillip sei wahrscheinlich im Jahre 1468 geboren. Demnach ist zu vermuten, dass Antoine de Fevin nicht lange nach Phillip geboren wurde, also zwischen Jahre 1470-1475. Antoine de Fevin stirbt sehr jung im Alter 35-40 Jahre. ${ }^{11}$

Andere Informationen aus seinem Leben sind schwer nachzuweisen. Wahrscheinlich studierte er an der Universität in Paris, denn Crétin nennt ihn als „maistre Antoyne Fenin“. ${ }^{12}$ Möglicherweise hatte Antoine an der Pariser Universität Magister Artium erworben. Anscheinend lernte er in Paris auch die königliche Kapelle kennen und trat den Dienst beim Ludwig XII. an. Trotz weniger Lebensangaben ist sicher, dass Antoine de Fevin sich großer Ruhm und Achtung seiner Zeitgenossen erfreute. Im Jahre 1515 verlegte nämlich Ottaviano Petrucci eine Kollektion seiner Messen. Pierre Moulu nannte ihn in seiner Mottete Mater floreat neben allen großen Komponisten dieser Zeit. Jean Mouton, seiner Kollege auf dem französischen Hof, schrieb anlässlich seines Todes ein Chanson „Qui ne regrettoit le gentil Févin?" ${ }^{13}$, in freier

5 KAHMANN, Ben. Antoine de Févin, a Biographical Contribution. In Musica disciplina, vol. 4, 1950 , s. 157. Übernommen aus PIRRO, A. Revue musicale, IV année, 1927, S. 252.

6 Zitiert nach KAHMANN, Ben. Antoine de Févin, a Biographical Contribution. In Musica disciplina, vol. 4, 1950, s. 157. Übernommen aus PEYROT, J. La tribune de St. Gervais, XXI année, 1919, S. 4-7 und 35-38.

7 Siehe KAHMANN, Ben. Antoine de Févin, a Biographical Contribution. In Musica disciplina, vol. 4, 1950, S. 157.

8 Ebenda, S. 143-155.

9 WALTER, Johann Gottfried. Musikalisches Lexicon. Leipzig: Wolfgang Deer, 1732.

10 AMBROS, August Wilhelm. Geschichte der Musik. 3. Band. Leipzig: F. E. C. Leuckart, 1881, S. 280.

11 KAHMANN, Ben. Antoine de Févin, a Biographical Contribution. In Musica disciplina, vol. 4, 1950, S. 160.

12 CRÉTIN, Guillaume. Plainte sur le trespas de feu maistre Jehan Braconnier, hrsg. von Kathleen Chesney, Paris 1932.

13 Zitiert nach SCHMIDT-BESTE, Thomas. Févin, Antoine de. In BLUME, Friedrich - FINSCHER, Ludwig. 
Übersetzung „Wer betrauert den liebenswürdigen Fevin?“ Und zuletzt selbst Glarean hielt ihn in seinem Musiktheoretischen Traktat für einen Nachfolger von Josquin Desprez. ${ }^{14}$ Diese Belege weisen eindeutig auf die Bedeutung von Antoine de Fevin innerhalb der dritten Generation der franko-flämischen Komponisten hin.

\section{Messen von Antoine de Fevin}

Die Menge der erhaltenen Kompositionen ist trotz weniger Personaldaten umfangreich. Was die Messen betrifft, handelt es sich um elf kompletten Messen und zwei teilweise überlieferten. Seit der Entdeckung vom Brünner Manuskript BAM1, ${ }^{15}$ gehört zu ihnen auch die Messe Helas ye suis mari. Aber wir betrachten in vielen Fällen Probleme mit einer richtigen Bestimmung der Attribution. Es steht also nicht fest, ob alle diese Messen zu Schaffen von Antoine de Fevin zugeordnet werden können.

\begin{tabular}{|l|l|}
\hline Name: & Vorlage: \\
\hline Missa de feria (5 $\mathrm{v})$ & Choral \\
\hline Missa de feria (4 $\mathrm{v})$ & $?$ \\
\hline Mente tota & Motette (Josquin) \\
\hline Ave Maria & Motette (Josquin) \\
\hline Sancta Trinitas & Motette (Fevin) \\
\hline O quam glorifica luce & Choral \\
\hline Requiem & Choral \\
\hline Salve Sancta parens & Choral \\
\hline Dictes moy & Chanson (Compére) \\
\hline Missa parva & Choral \\
\hline Sanctorum meritis & Choral \\
\hline Missa (ohne Name) & nicht komplett \\
\hline Missa ad placitum & nicht komplett \\
\hline Missa Helas ye suis mari & Chanson (Fevin) \\
\hline
\end{tabular}

Tab. 1. Antoine de Fevins Messen

Die Musik in Geschichte und Gegenwart: Allgemeine Enzyklopädie der Musik. 21 Bände in zwei Teilen. 2. neubearb. Ausg. Kassel: Bärenreiter, 2001, S. 1104.

14 GLAREAN, Heinrich. Dodecachordon. Basel, 1547, S. 354.

15 Bam1 (Archiv der Stadt Brünn. Handschriftensammlung, Sig. 15/4). Dieses Manuskript wurde erstmal von Martin Horyna und Vladimír Maňas beschreiben (HORYNA, Martin - MAŇAS, Vladimír. Two Manuscripts of Polyphonic Music in Brno from the Mid-Sixteenth Century. Early Music 40, 2012, Nr. 4, S. 553-575). Weitere Informationen darüber bringen auch an der Masaryk Universität entstandene Diplomarbeiten: (POLÁČEK, Radek. Brünner Manuskript Liber Missarum aus dem Jahre 1550 und da erhaltene Messen von Wolfgang Gräfinger. Bachelorarbeit. Institut für Musikwissenschaft, Philosophische Fakultät, Masaryk Universität, Brünn 2009; POLÁČEK, Radek. Heinrich Finck und Thomas Stolzer Messen im Brünner Manuskript. Masterarbeit, Institut für Musikwissenschaft, Philosophische Fakultät, Masaryk Universität, Brünn 2011; MILJKOVIĆ, Đorđe. Missa Matthei Parthenii und ihre Notenschrift- und Transkriptionsproblematik. Bachelorarbeit. Institut für Musikwissenschaft, Philosophische Fakultät, Masaryk Universität, Brünn 2011; STUDENIČOVÁ, Hana. Antoine a Robert de Fevin und ihre Messen im Brünner Manuskript BAM1. Masterarbeit, Institut für Musikwissenschaft, Philosophische Fakultät, Masaryk Universität, Brünn 2015). 
Das Werk von Antoine de Fevin fällt in ein historisches Zeitalter der Änderungen, in dem eine Gleichberechtigung aller Stimmen als Träger des imitativen Kontrapunkts und auch des Textes vorkommt. Vierstimmigkeit herrscht bereits bei Dufay vor (der ein Vertreter der zweite Generation der franko-flämischen Komponisten war) und wird bis etwa 1520 relativ selten über- oder unterschritten. ${ }^{16}$ Nächste Generation der Komponisten erhöhte die Zahl der Stimmen. Noch bei Josquin und seinen Zeitgenossen ist Mehrheit der Messen vierstimmig, aber schon häufiger auch fünf- oder sechsstimmig. Bei Fevin herrscht nach dem Vorbild der zeitgemäßen Konventionen Vierstimmigkeit vor. Die einzige Messe für fünf Stimmen heißt Missa de feria. Andere Situation ist in Missa pro fidelibus defunctis (Requiem) zu sehen. Dieses Requiem ist vierstimmig, aber in Sätzen Sanctus und Agnus Dei stieg der Anzahl der Stimmen (noch zweite Bassus), sie sind also für fünf Stimmen. Die Steigerung der Stimmenanzahl am Ende der Messe symbolisiert eine damals nicht ungewöhnliche Gradation der ganzen Komposition. ${ }^{17}$

Fevin mit seinen Kollegen am französischen Hof (Divitis und Mouton) befestigt die Tradition der Parodiemesse, die seine Vorgänger einführte. ${ }^{18}$ Üblicherweise komponierte Antoine de Fevin seine Messen mit einem parodischen Prinzip. Als Zitate nutzte er Teile aus sakralen und weltlichen Stücken.

\section{Missa Ave Maria}

Die Messe Ave Maria, die im BAM1 überliefert ist, gehört zu meist verbreiteten Messen der Komponist Antoine de Fevin. Dies passierte hauptsächlich dank des Messendrucks von Petrucci aus dem Jahr $1515 .{ }^{19}$ In dem Fall Missa Ave Maria komponierte er mit dem parodischen Prinzip, in dem er die Motette Ave Maria von Josquin Desprez zitierte.

Insgesamt gibt es sieben Manuskripte, in denen Missa Ave Maria komplett erhalten ist. Weitere vier Versionen sind nur fragmentarisch überliefert.

\begin{tabular}{|l|l|}
\hline Kürzel: & Besitzer: \\
\hline Brno AMB 15/4 & $\begin{array}{l}\text { Brno, Archiv města Brna. Sbírka rukopisů, fond V 2 Svatojakubská knihovna, } \\
\text { sign.15/4 (Brünn, Archiv der Stadt Brünn. Handschriftensammlung, Bestand V2 } \\
\text { St.-Jakobs-Bibliothek, Sig. 15/4) }\end{array}$ \\
\hline D-HRD & Herdringen, Schloss Fürstenberg, Bibliothek, Ms. 9820 \\
\hline Fr2 & Frankfurt, Stadt- und Universitäts- Bibliothek, Mus. Hs. fol. -2. \\
\hline Jena U7 & Jena, Thüringer Universitäts- und Landesbibliothek, MS 7 \\
\hline
\end{tabular}

16 KAST, Paul. Die mehrstimmige Messe. In Musikalische Gattungen in Einzeldarstellungen. Band 2 - Die Messe. München: dtv, 1985, S. 46.

17 Ebenda, S. 46.

18 SCHMIDT-BESTE, Thomas. Févin, Antoine de. In BLUME, Friedrich - FINSCHER, Ludwig. Die Musik in Geschichte und Gegenwart: Allgemeine Enzyklopädie der Musik. 21 Bände in zwei Teilen. 2. neubearb. Ausg. Kassel: Bärenreiter, 2001, S. 1102.

19 RISM 1515 ${ }^{1}$; PETRUCCI, Ottaviano. Antoine de Fevin [et al.]: Misse. Fossombrone, 1515, [online]. Letzter Zugriff am 5. Mai 2015, 〈http://imslp.nl/imglnks/usimg/f/fa/IMSLP277134-PMLP449965-petrucci_misse.pdf>. 
Antoine und Robert de Fevin: Ihre Messen in Brünner Manuskript BAM1

\begin{tabular}{|l|l|}
\hline Kürzel: & Besitzer: \\
\hline St 45 & $\begin{array}{l}\text { Stuttgart, Württembergische Landesbibliothek, } \\
\text { MS Musicale folio I 45 }\end{array}$ \\
\hline Tu59 & Turin, Biblioteca Nazionale Universitaria, Cod. Q. m. III 59 \\
\hline Upps U 76c & Uppsala, Universitetsbiblioteket, MS Vokalmusik i Handskrift 76c \\
\hline VatG XII. 2 & Roma, Biblioteca Apostolica Vaticana, MS Cappella Giulia XII. 2 \\
\hline Vat CS 45 & Roma, Biblioteca Apostolica Vaticana, Cappella Sistina 45 \\
\hline Vat SMM & Roma, Biblioteca Apostolica Vaticana, Santa Maria Maggiore JJ. III. 4 \\
\hline 1545/6 & Bicinia gallica, latina, germanica - Tomus primus (Wittenberg, 1545) \\
\hline
\end{tabular}

Tab. 2. Missa Ave Maria - die Manuskripte

Der Druck von Petrucci erschien nach dem Tod von Antoine Fevin und außer seinen drei Messen (Ave Maria, Mente tota und Missa Sancta trinitas) beinhaltet er auch die Messe Le vilayn ialoys von Robert de Fevin und die Messe Quarti toni von Pierzon (Pierre de la Rue).

Auch andere bekannte Drucker gaben Antoines Werke heraus, die ihre Popularität beweist. Zu dem größten Konkurrent Petruccis gehörte ohne Zweifel Andrea Antico aus Rom, der im Jahr 1516 einen Band mit fünfzehn Messen verlegte, in dem gerade drei Messen von Antoine de Fevin (Missa Ave maria, Mente tota und Missa de feria) enthalten sind. ${ }^{20}$ Nach diesen zwei Drucken erschienen nächsten 15 Jahren keine weiteren Kompositionen von Antoine de Fevin.

\begin{tabular}{|l|l|l|l|}
\hline Drucker: & Name: & Ort: & Jahr: \\
\hline Antico, Andrea & $\begin{array}{l}\text { Liber quindecem missarum electarum que per } \\
\text { excellentissimos musicos compositae fuerunt }\end{array}$ & Rom & 1516 \\
\hline Gardane, Antonio & Il primo libro a due voci de diversi autori & Venedig & 1543 \\
\hline $\begin{array}{l}\text { Montanus \& } \\
\text { Neuber }\end{array}$ & $\begin{array}{l}\text { Diphona amoena et florida, selectore Erasmo } \\
\text { Rotenbuchero, boiaro. }\end{array}$ & Nürnberg & 1549 \\
\hline $\begin{array}{l}\text { Petrucci, } \\
\text { Ottaviano }\end{array}$ & Misse Antonii de Fevin [et al.] & Fossombrone & 1515 \\
\hline Scotto, Girolamo & $\begin{array}{l}\text { Intabulatura di lautto, libro quarto de la messa di A. } \\
\text { Fevino, sopra Ave Maria }\end{array}$ & Venedig & 1546 \\
\hline
\end{tabular}

Tab. 3. Missa Ave Maria - Die Drucke

\section{Missa Helas ye suis mari}

Was die Messe Helas ye suis mari betrifft, vermissen wir in diesem Moment solche breite Quellenbasis, weil jene Messe in Brünner Manuskript BAM1 wahrscheinlich einmalig

20 ANTICO, Andrea. Liber quindecim missarum. Rome, 1516, [online]. Letzter Zugriff am 3. Mai 2015, 〈http://conquest.imslp.info/files/imglnks/usimg/d/dd/IMSLP307724-PMLP497646-bologna.pdf〉. 
überliefert ist. ${ }^{21}$ Wenn sich nicht der Schreiber verschrieben hatte, geht es wirklich um eine weitere Messe von Antoine de Fevin, weil die Atributtionsbestimmung sehr klar zu sehen ist. Die Autorschaft der Messe unterstützt die Tatsache, dass Antoine de Fevin ein gleichnamiges Chanson komponierte. Die ausführliche Analyse der Messe bestätigte, dass er sein Chanson mit parodischem Prinzip benutzte. In diesem Fall sehen wir eine bedeutende Verschiebung in bisheriger Forschung.

\section{Robert de Fevin: Bruder, Verwandte oder Fremde?}

Ganz andere Situation tritt bei Robert de Fevin ein. Über Robert kann mit Sicherheit gesagt werden, dass seine Messe Le vilayn ialoys Ottaviano Petrucci in Messekollektion von Antoine de Fevin gedruckt wurde. ${ }^{22}$

Robert de Fevin war ein aktiver Musiker am Anfang des 16. Jahrhunderts. In Lexiken schreibt über ihn als erster François-Joseph Fétis. Er berichtet, dass Robert aus Cambrai kam und der Kapellmeister bei dem savoyischen Herzog war (wahrscheinlich in Chambéry). ${ }^{23}$

Auch in Brockhaus Riemann Musiklexikon ist er erwähnt, wovon der letzte Satz der wichtigste ist: „Über eine verwandtschaftliche Verbindung mit Antoine de Fevin ist nichts bekannt ". ${ }^{24}$ Es ist schwierig zu beweisen, dass Antoine und Robert Verwandte waren, geschweige denn Brüder. In dem Stammbaum figuriert Roberts Name gar nicht. ${ }^{25}$ Dort stehen nur Namen Philippe und Antoine geschrieben. Einziger Nachweis, der sein Verwandtschaftsverhältnis zugibt, befindet sich in der bekannten Motette Mater floreat des Komponist Pierre Moulu. ${ }^{26}$ Der bekannte Musiker nannte vierundzwanzig Namen der berühmten Komponisten der zweiten und dritten Generation. Moulu nennt Komponisten wie Dufay, Busnoys, Obrecht, Compére, De la Rue, weiter auch Komponisten, die zu der französischen königlichen Kapelle gehören (Prioris, Divitis, Mouton, Fevin, Hilaire). Außerdem durften die Namen Josquin, Isaac, Brumel und anderen nicht fehlen. ${ }^{27}$

21 Bam1 (Archiv der Stadt Brünn. Handschriftensammlung, Sig. 15/4).

22 PETRUCCI, Ottaviano. Antoine de Fevin [et al.]: Misse. Fossombrone, 1515, [online]. Letzter Zugriff am 5. Mai 2015, 〈http://imslp.nl/imglnks/usimg/f/fa/IMSLP277134-PMLP449965-petrucci_misse.pdf〉.

23 Nach François-Joseph Fétis befinden sich diese Informationen in Monumenta patriae, Turino, etwa 1843. Es ist mir nicht gelungen, diese Angabe von Fétis zu überprüfen. Über dieses Problem mit der Fevins Zitation schreibt mehr CLINKSCALE, Edward Henry. Masses and Motet von Robert de Fevin. Ottawa, Canada: Institute of Mediaeval Music, 1993, S. 12.

24 DAHLHAUS, Carl - EGGEBRECHT, Hans Heinrich. Fevin, Antoine de. In Brockhaus Riemann Musiklexikon. Erster Band. Wiesbaden, 1978, S. 403.

25 PUISIEUX, G. B. de. Pierre de Févin, chronique artésiem du XVe siécle. In Bulletin de la Société des antiquaires de Picardie, 1909-1910, S. 325.

26 FALLOWS, David. Josquin. Turnhout: Brepols, 2009, S. 309-310. Zitiert nach LOWINSKY, Edward. The Medici Codex. 1957 a 1968. Für weitere Informationen siehe auch WEXLER, Richard. Antoine Bruhier. Life and Works of Renaissance Papal Composer. Brepols Pub, 2014.

27 FALLOWS, David. Josquin. Turnhout: Brepols, 2009, S. 309-310. 
In einem Vers dieser Motette steht: „Nec absint decori fratres de Fevin“, frei übersetzt „die Brüder Fevins dürfen nicht fehlen“. Dass die Namen Fevins auch hier neben allen bedeutenden Komponisten erscheinen, beweist anscheinend ihre Fähigkeiten und große Anerkennung beider Komponisten in dieser Zeit.
$[\ldots]$
Lourdault; Prioris amenus.
Nec absint decori fratres de Fevin,
Hilaire hilaris, Divitis felix,
Brumel, Isaac, Nynot,
Mathurin Forestier, Bruhier facundi,
Mouton cum vellere aureo.
$[\ldots]^{28}$

Das Datum von Roberts Tod wird nur aus der Zuschrift „Robertus de fevin pie memorie“ bei seiner Messe La sol mi fa re abgeschätzt. ${ }^{29}$ Diese Messe wurde in Münchner Manuskripts Nummer 7 erhalten, das in Zeitraum der Jahre $1512-1530$ datiert ist. ${ }^{30}$ Zu vermuten ist, dass Robert de Fevin in diesen Jahren schon verstorben war.

\section{Kompositionsstil und Widersprüche der Autorschaft}

Nicht nur wenige Lebensinformationen, sondern auch nicht viel erhaltenen Kompositionen ziehen die Existenz des Komponist Robert de Fevin in Zweifel.

Der Werke von Antoine und Robert de Fevin widmete seine Aufmerksamkeit kanadischer Musikwissenschaftler Edward Clinkscale und edierte alle ihre bis zu diesem Zeitpunkt bekannten Kompositionen. ${ }^{31}$ Er ordnete Robert wegen seines konservativeren Kompositionsstils zur späteren zweiten Generation, wobei Antoine de Fevin zu der dritten hingehört. In Roberts Kompositionen können wir den Erfindergeist vermissen, der für Antoine so typisch ist. Bei der Vermutung, dass Roberts Schaffen zur dritten Generation gehört, muss in Betracht gezogen werden, dass sich seine Kompositionstechnik dem Josquin verzögert und noch entfernter von den Komponisten auf dem königlichen Hof ist. ${ }^{32}$ Daraus ergibt sich, dass sich Robert de Fevin an der Grenze der zweiten und dritten

\footnotetext{
28 FALLOWS, David. Josquin. Turnhout: Brepols, 2009, S. 310.

29 MunBs7 (München, Bayerische Staatsbibliothek, Musiksammlung, Musica MS 7). Siehe ClinksCale, Edward Henry. Masses and Motet von Robert de Fevin. Ottawa, Canada: Institute of Mediaeval Music, 1993, S. 11.

30 Mehr Informationen siehe auch in CLINKSCALE, Edward Henry. Masses and Motet von Robert de Fevin. Ottawa, Canada: Institute of Mediaeval Music, 1993.

31 CLINKSCALE, Edward Henry. The Complete Works of Antoine de Févin. Diss., New York University, 1965; CLINKSCALE, Edward Henry. Masses and Motet von Robert de Fevin. Ottawa, Canada: Institute of Mediaeval Music, 1993.

32 CLINKSCALE, Edward Henry. Masses and Motet von Robert de Fevin. Ottawa, Canada: Institute of Mediaeval Music, 1993, S. 12.
} 
Generation der franko-flämischen Polyphonie bewegt. Neuerungen sind bei ihm in der Arbeit mit Cantus firmus zu sehen, aber nur am Anfang der Messe Ave Maria und am Ende der Messe Le vilayn ialoys. Beide genannten sind die zwei von drei komplett erhaltenen Messen von Robert de Fevin. Alle seine drei Messen sind vierstimmig komponiert.

\begin{tabular}{|l|l|}
\hline Name: & Vorlage: \\
\hline Missa Le vilayn ialoys & Motette (Josquin Desprez) \\
\hline Missa Ave Maria & Motette (Josquin Desprez) \\
\hline Missa La sol mi fa re & $?$ \\
\hline Credo La belle se seit & Chanson (Dufay) \\
\hline
\end{tabular}

Tab. 4. Messe von Robert de Fevin

Im Falle Missa Le vilayn ialoys, der Ottaviano Petrucci im Jahre 1515 druckte, sind nur zwei Manuskripte bekannt, in denen sich diese Messe befindet. Missa Le vilayn ialoys ist in Brünner Manuskript BAM1 erhalten. ${ }^{33}$ Das zweite Manuskript heißt Manuskript Cappella Sistina 23, aber es gibt dort keine Autorschaftsangabe („Anonym“). ${ }^{34}$

\begin{tabular}{|l|l|}
\hline Kürzel: & Besitzer: \\
\hline VatS 45 & Roma, Biblioteca Apostolica Vaticana, Cappella Sistina 45 \\
\hline Brno AMB 15/4 & $\begin{array}{l}\text { Brno, Archiv města Brna. Sbírka rukopisů, fond V 2 Svatojakubská knihovna, sign. } \\
\text { 15/4 (Brünn, Archiv der Stadt Brünn. Handschriftensammlung, Bestand V2 St.- } \\
\text { Jakobs-Bibliothek, Sig. 15/4) }\end{array}$ \\
\hline
\end{tabular}

Tab. 5. Missa Le vilayn ialoys - die Manuskripte

Ich habe festgestellt, dass die Vorlage der Missa Le vilayn ialoys, die in Brünner Manuskript BAM1 erhalten ist, Josquins Komposition Le villain bildet. Diese Komposition ist ohne Text überliefert. ${ }^{35}$

In Missa Ave Maria, wie in anderen Kompositionen von Robert de Fevin, betrachten wir Probleme mit einer richtigen Bestimmung der Attribution. Die Messe Ave Maria komponierte nämlich sowohl Robert, als auch Antoine. Das wäre an sich nichts Außerordentliches. Überraschend ist aber die Tatsache, dass dieselbe Mottete Ave Maria von Josquin Desprez die Vorlage der beiden Messen Ave Maria (sowohl von Robert als auch

33 BAM1 (ARchiv mĚsta Brna. Sbírka RUkopisư, Sign. 15/4).

34 CLINKSCALE, Edward Henry. Masses and Motet von Robert de Fevin. Ottawa, Canada: Institute of Mediaeval Music, 1993, S. 17.

35 Diese Komposition befindet sich in der Musikhandschrift 2 Codex 142A Der StaAts- und StadtbiblioTher Augsburg. Die Editionen: DESPREZ, Josquin. Le villain. [online]. Letzter Zugriff am 10. Oktober 2015, <http://imslp.org/wiki/Le_villain_(Josquin_Desprez)>; JONAS, Luise. Das Augsburger Liederbuch: die Musikhandschrift $2^{\circ}$ Codex 142 a Der Staats- und Stadtbibliothek Augsburg: Edition und Kommentar. München: Musikverlag Emil Katzbichler, 1983. I., Notenband, S. 121-124; II. Textband, S. 145-146; FALLOWS, David [Hrsg.]. The collected works of Josquin des Prez vol. 28. Secular works for four voices. Partitur. 2005, S. 57. 
von Antoine) darstellt. Weiterer Widerspruch der Autorschaft ist möglich in Lamentationes hieremie zu sehen. Dieses Werk ist beiden Komponisten zuzuschreiben. Suspekt ist, dass sich in allen Manuskripten, in denen die Messen von Robert de Fevin überliefert sind, die Kompositionen auch von Antoine de Fevin befinden. ${ }^{36}$

Eine ähnliche Unstimmigkeit kommt zum Vorschein bei einem eigenständig vertonten Teil Credo für vier Stimmen. Die Vorlage von Credo ist Chanson La belle se seit von Guillaume Dufay. Dieses Patrem druckte Ottaviano Petrucci im Jahre 1505 in Fragmentum missarum und gehört zu Josquin. ${ }^{37}$ Jenes Credo ist im Kodex Nummer 41 von Manuskript Cappella sistina in Vatikan erhalten. ${ }^{38}$ Das Credo ist aber in diesem Manuskript dem Robert de Fevin zugeschrieben. Wie Edward Clinkscale schrieb, Credo La belle se seit ist entweder sehr hochentwickeltes Beispiel des Kompositionsstils von Robert de Fevin, oder im Gegenteil nicht so gelungene Komposition von Josquin Desprez. ${ }^{39}$

Angesichts der wenigen erhaltenen Informationen war auch die Möglichkeit abgewogen, dass die Existenz des Komponisten Robert de Fevin nur Folge der falschen Attributionen ist. Aber wie schon gesagt wurde, Antoine und Robert komponierten in einem anderen Kompositionsstil. Infolgedessen scheint die Hypothese, dass es um dieselbe Person geht, eher unwahrscheinlich. Aufgrund der mangelnden Quellen ist es jedoch unmöglich diesen Widerspruch zu klären.

Zusammenfasend lässt sich sagen, dass alle drei Messen von Antoine und Robert de Fevin, die in Brünner Manuskript BAM1 erhalten sind, einen Beweis für die Entwicklung des Kompositionsstils binnen zwei Generationen der franko-flämischen Polyphonie darstellen. Während Missa Le vilayn ialoys Ende der zweiten Generation vertritt, gehören die Messen von Antoine de Fevin (Ave Maria und Helas ye suis mari) mit ihren Elementen in die dritte Generation. Dank dieser Forschung konnte die Wichtigkeit beiden Komponisten im mitteleuropäischen Kontext nachgewiesen werden.

\section{Bibliography}

AMBroS, August Wilhelm. Geschichte der Musik. 3. Band. Leipzig: F. E. C. Leuckart, 1881, S. 279-281.

CLINKSCALE, Edward Henry. The Complete Works of Antoine de Févin. Diss., New York University, 1965.

CLINKSCALE, Edward Henry. Masses and Motet von Robert de Fevin. Ottawa, Canada: Institute of Mediaeval Music, 1993.

36 Zum Beispiel: Manuskript Santa Maria Maggiore 26; VatS 26 (Roma, Biblioteca Apostolica Vaticana, MS Cappella Sistina 26); VatSM 26 (Roma, Biblioteca Apostolica Vaticana, MS Santa Maria Maggiore 26) und andere.

37 RISM 1505' ${ }^{1}$; PETRUCCI, Ottaviano. Fragmenta missarum. Venice, 1505, [online]. Letzter Zugriff am 7. April 2015, <http://burrito.whatbox.ca:15263/imglnks/usimg/4/4f/IMSLP306900-PMLP496451-petrucci_fragmenta_missarum.pdf $>$.

38 VatS 41 (Roma, Biblioteca Apostolica Vaticana, MS Cappella Sistina 41).

39 CLINKSCALE, Edward Henry. Masses and Motet von Robert de Fevin. Ottawa, Canada: Institute of Mediaeval Music, 1993, S. 13. 
CRÉTIN, Guillaume. Plainte sur le trespas de feu maistre Jehan Braconnier, hrsg. von Kathleen Chesney, Paris 1932.

DAHLHAUS, Carl - EGGEBRECHT, Hans Heinrich. Fevin, Antoine de. In Brockhaus Riemann Musiklexikon. Erster Band. Wiesbaden, 1978, S. 403.

DELPORTE, Julius. Antoine de Févin (147.-1512). In Revue liturgique et musicale, XVIII. 1934-1935, S. 27-31; 42-44; 54-57; 75.

EGGEBRECHT, Hans Heinrich. Musik im Abendland: Prozesse und Stationen vom Mittelalter bis zur Gegenwart. München: Piper, 1991.

FALLOWS, David. Josquin. Turnhout: Brepols, 2009, S. 309-310.

FALLOWS, David [Hrsg.]. The collected works of Josquin des Prez vol. 28. Secular works for four voices. Partitur. 2005, S. 57.

FÉTIS, François-Joseph. Fevin, Antoine. In Biographie universelle des musiciens. Second Edition. Paris: Firmin Didot Fréres, 1866, S. 241-242.

FÉTIS, François-Joseph. Fevin, Robert. In Biographie universelle des musiciens. Second Edition. Paris: Firmin Didot Fréres, 1866, S. 242.

GLAREAN, Heinrich. Dodecachordon. Basel, 1547.

HORYNA, Martin - MAŇAS, Vladimír. Two Manuscripts of Polyphonic Music in Brno from the Mid-Sixteenth Century. Early Music 40, 2012, N. 4, S. 553-575.

JONAS, Luise. Das Augsburger Liederbuch: die Musikhanschrift $2^{\circ}$ Codex $142 a$ Der Staats- und Stadtbibliothek Augsburg: Edition und Kommentar. München: Musikverlag Emil Katzbichler, 1983.

KAHMANN, Ben. Antoine de Févin, a Bio-Bibliographical Contribution. In Musica disciplina, vol. 4, 1950, S. 153-162; vol. 5, 1951, S. 143-155.

KAST, Paul. Die mehrstimmige Messe. In Musikalische Gattungen in Einzeldarstellungen. Band 2 Die Messe. München: dtv, 1985.

MILJKOVIĆ, Đorđe. Missa Matthei Parthenii und ihre Notenschrift- und Transkriptionsproblematik. Bachelorarbeit. Institut für Musikwissenschaft, Philosophische Fakultät, Masaryk Universität, Brünn 2011.

POLÁČEK, Radek. Brünner Manuskript Liber Missarum aus dem Jahre 1550 und da erhaltene Messen von Wolfgang Gräfinger. Bachelorarbeit. Institut für Musikwissenschaft, Philosophische Fakultät, Masaryk Universität, Brünn 2009.

POLÁČEK, Radek. Heinrich Finck und Thomas Stolzer Messen im Brünner Manuskript. Masterarbeit, Institut für Musikwissenschaft, Philosophische Fakultät, Masaryk Universität, Brünn 2011.

PUISIEUX, G. B. de. Pierre de Févin, chronique artésiem du XVe siécle. In Bulletin de la Société des antiquaires de Picardie, 1909-1910, S. 322-333.

SCHMIDT-BESTE, Thomas. Févin, Antoine de. In BLUME, Friedrich - FINSCHER, Ludwig: Die Musik in Geschichte und Gegenwart: Allgemeine Enzyklopädie der Musik. 21 Bände in zwei Teilen. 2. neubearb. Ausg. Kassel: Bärenreiter, 2001, S. 1102.

STUDENIČOVÁ, Hana. Antoine a Robert de Fevin und ihre Messen im Brünner Manuskript BAM1. Masterarbeit, Institut für Musikwissenschaft, Philosophische Fakultät, Masaryk Universität, Brünn 2015.

WALTER, Johann Gottfried. Musikalisches Lexicon. Leipzig: Wolfgang Deer, 1732. 
